\title{
Cutaneous microsporidiosis in an immunosuppressed patient
}

\author{
Daniel A. Nadelman BA ${ }^{1}$ ( ) | Ashley R. Bradt DO ${ }^{2} \quad$ Yvonne Qvarnstrom PhD ${ }^{3}$ | \\ Cynthia S. Goldsmith MGS ${ }^{4}$ | Sherif R. Zaki MD, PhD ${ }^{4}$ Frank Wang MD ${ }^{5}$ | \\ Emily H. Smith $M^{6}$ | Douglas R. Fullen $M^{2,5}$
}

${ }^{1}$ University of Michigan Medical School, Ann Arbor, Michigan, USA

${ }^{2}$ Department of Pathology, University of

Michigan, Ann Arbor, Michigan, USA

${ }^{3}$ Centers for Global Health, Division of Parasitic Diseases, Centers for Disease Control and Prevention (CDC), Atlanta, Georgia

${ }^{4}$ National Center for Emerging and Zoonotic Infectious Diseases, Division of High-

Consequence Pathogens and Pathology,

Centers for Disease Control and Prevention

(CDC), Atlanta, Georgia

${ }^{5}$ Department of Dermatology, University of

Michigan, Ann Arbor, Michigan, USA

${ }^{6}$ Department of Dermatology, University of

Missouri, Columbia, Missouri, USA

Correspondence

Douglas R. Fullen, MD, Department of

Pathology, Michigan Medicine, NCRC, Bldg.

35, 2800 Plymouth Road, Ann Arbor, MI

48109, USA.

Email: dfullen@med.umich.edu

\begin{abstract}
Microsporidia are a group of obligate intracellular parasites that naturally infect domestic and wild animals. Human microsporidiosis is an increasingly recognized multisystem opportunistic infection. The clinical manifestations are diverse with diarrhea being the most common presenting symptom. We present a 52-year-old woman with a history of amyopathic dermatomyositis complicated by interstitial lung disease managed with mycophenolate mofetil and hydroxychloroquine who presented with a 7-month history of recurrent subcutaneous nodules as well as intermittent diarrhea and chronic sinusitis. A punch biopsy showed superficial and deep lymphocytic and granulomatous dermatitis with focal necrosis. Tissue stains for microorganisms revealed oval 1 to $3 \mu \mathrm{m}$ spores within the necrotic areas in multiple tissue stains. Additional studies at the Centers for Disease Control and Prevention confirmed cutaneous microsporidiosis. This case is one of very few confirmed examples of cutaneous microsporidiosis reported in the literature.
\end{abstract}

\section{KEYWORDS}

cutaneous, immunosuppression, microsporidia, microsporidiosis

\section{1 | INTRODUCTION}

Microsporidia are a group of obligate intracellular organisms, which typically cause limited intestinal disease in immunosuppressed patients, especially in those with advanced human immunodeficiency virus/acquired immunodeficiency syndrome (HIV/AIDS). The extraintestinal manifestations of microsporidiosis are diverse and include cardiac, pulmonary, ocular, and renal disease..$^{1-5}$

Microsporidia were previously considered protozoa but have since been reclassified as fungi based on spore size, nucleus arrangement, mode of replication, and rRNA analysis. ${ }^{2,5}$ Over 1200 species have been identified, 15 of which have been reported as human pathogens. ${ }^{6}$ The most common species infecting humans is Enterocytozoon bieneusi, followed by the Encephalitozoon species (particularly Encephalitozoon intestinalis). Enterocytozoon infections normally cause localized infection; in contrast, Encephalitozoon species have the ability to disseminate widely via macrophages, thus causing systemic infection involving the intestinal and hepatobiliary tracts, respiratory tract, sinuses, kidney, eye, brain, and skin. ${ }^{2,5,6}$ Anncaliia algerae has also been implicated in infections of the eye, skeletal muscle, and skin of humans. ${ }^{6}$

Transmission is not completely understood, but is thought to occur primarily by ingestion or inhalation of spores. ${ }^{1,5}$ Diagnosis can be achieved via light microscopy, transmission electron microscopy (TEM), immunofluorescence and immunohistochemical assays with polyclonal or monoclonal antibodies, cell culture, and polymerase chain reaction (PCR). ${ }^{2,3,7-9}$ Intestinal biopsy reveals distorted villous architecture, including blunting and hyperplasia of crypts and lack of an inflammatory response. ${ }^{1,2}$ Cutaneous involvement in disseminated microsporidiosis presents as painful, erythematous, scaly, and crusted skin nodules. Skin biopsy showed a dense dermal acute and granulomatous inflammatory reaction with presence of many spores. ${ }^{3}$ Erythematous macules and papules with histopathologic confirmation of involvement by microsporidia has also been reported 
in an immunocompromised patient. ${ }^{4}$ Spores may be highlighted with a number of different stains, including the modified trichrome/chromotrope 2R, acid-fast, periodic acid-Schiff, and Warthin-Starry
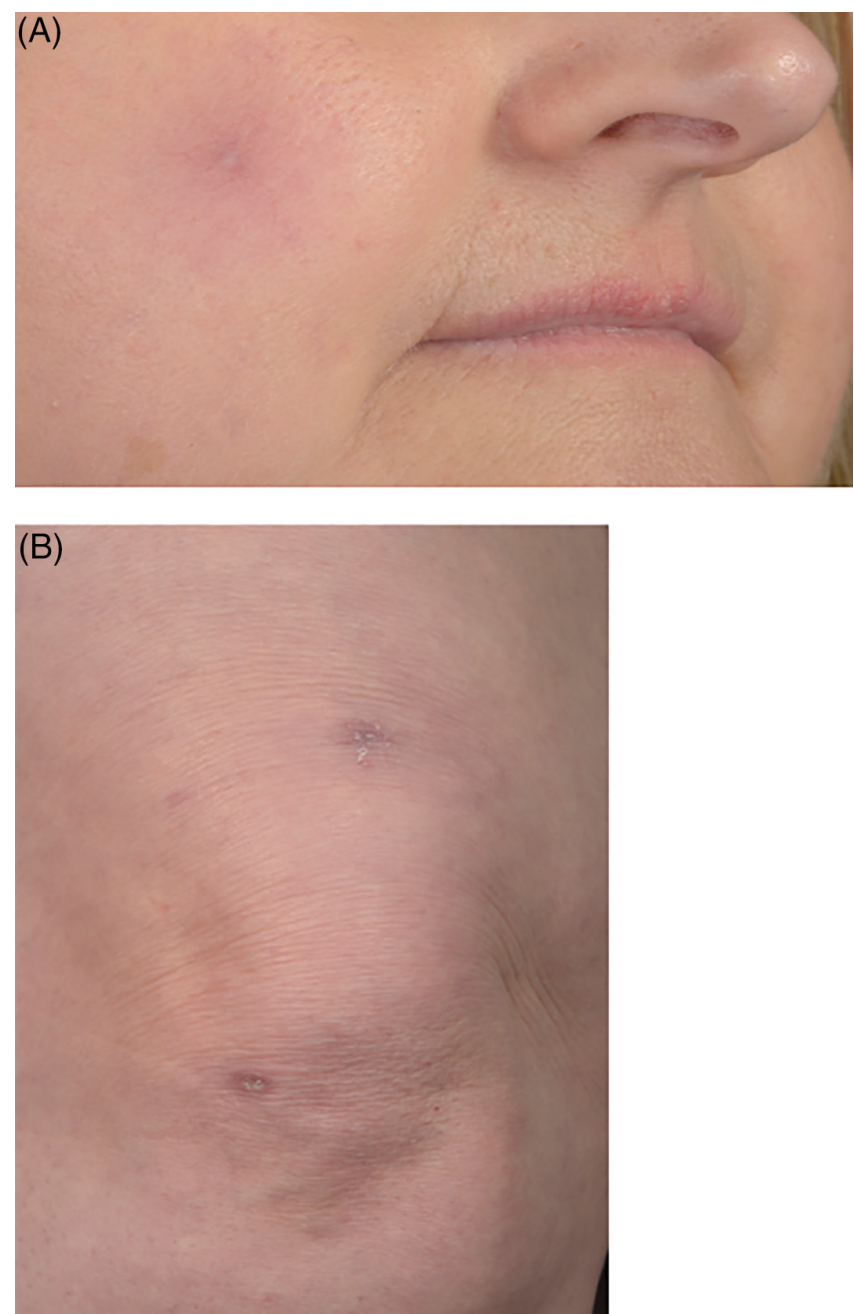

FIGURE 1 A and B, Diffuse flesh-colored subcutaneous nodules. Note the subtle overlying erythema of an early subcutaneous nodule in $A$, while $B$ reveals the older lesions with central hyperkeratotic plugs stains. ${ }^{7}$ These stains can be used on stool, urine, and respiratory specimens. TEM can identify substructural characteristics, such as number of polar filament cross sections, arrangement of polar filaments, and characteristics of the parasite-host interface, to differentiate among species. ${ }^{5}$

\section{2 | CASE REPORT}

A 52-year-old, HIV-negative female from rural Indiana presented with a 7-month history of recurrent subcutaneous nodules on her face, arms, and legs. Her past medical history was significant for amyopathic dermatomyositis complicated by interstitial lung disease, which was managed with mycophenolate mofetil and hydroxychloroquine. On examination, the lesions appeared as 1 to $1.5 \mathrm{~cm}$ subcutaneous flesh-colored nodules distributed diffusely. Some lesions had an erythematous-violaceous hue, and older lesions exhibited a central hyperkeratotic plug (Figure 1).

Previous biopsies on two separate occasions (January and April 2016), which were interpreted at another facility, showed a lymphoid infiltrate consistent with cutaneous lymphoid hyperplasia in the initial biopsy, and a necrotizing vasculitis involving medium-sized vessels with associated granulomatous inflammation in the subsequent biopsy. At that time, Giemsa and Fite stains were negative for infectious microorganisms. As the differential included granulomatosis with polyangiitis, the patient was treated with a 6-week prednisone taper, with initial mild improvement of the skin lesions. A review of those biopsies in our institution showed presence of microorganisms consistent with microsporidia in the biopsy from April 2016 but not in the biopsy from January 2016. The vasculitis evident in the April 2016 biopsy was subsequently regarded as a secondary phenomenon to the infectious process rather than a primary vasculitis because vasculitis has not been reported in localized or disseminated microsporidiosis to our knowledge.

Her skin nodules recurred once again, and punch biopsies were taken from nodules on the right lateral leg and left posterior leg in our institution in June 2016. Hematoxylin and eosin stain showed a
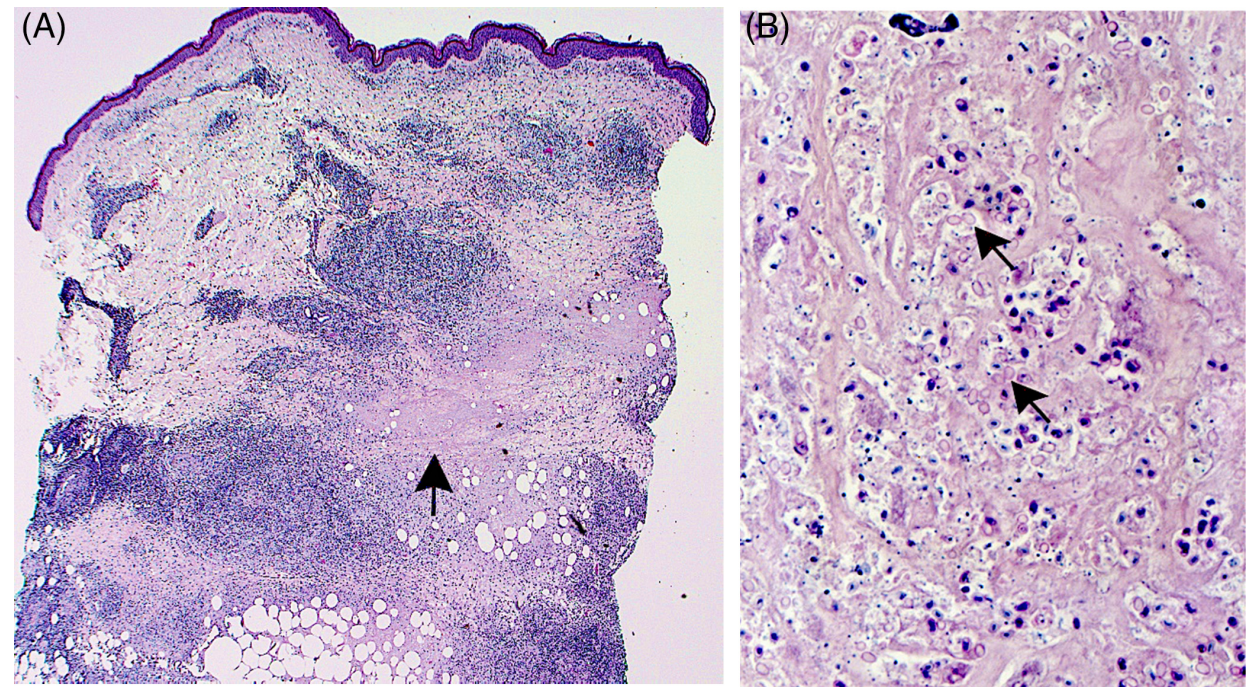

FIGURE 2 A and B, Biopsy from the left posterior leg. Lymphocytic infiltration within the dermis and subcutaneous tissues. Note the area of focal necrosis within the dermis (arrow), A, in which microorganisms consistent with microsporidia were present (arrows), B, Hematoxylin and eosin (H\&E). A, original magnification $\times 2.5$, $B$, original magnification $\times 100$ 
FIGURE 3 A-D, Tissue stains for microorganisms. A, Gomori methenamine silver (GMS) stain showing Anncaliia algerae spores (arrow) (original magnification $\times 100$ ). B, Immunohistochemical staining of spores (red) in same area shown in Figure 2A; naphthol fast red substrate with hematoxylin counterstain (original magnification $\times 100)$. C, L-T Gram stain highlights Gram-positive spores (arrow) (original magnification $\times 100$ ). $D$, Polarization of the same field in (C) showing bright white spores (arrow) (original magnification $\times 100$ )
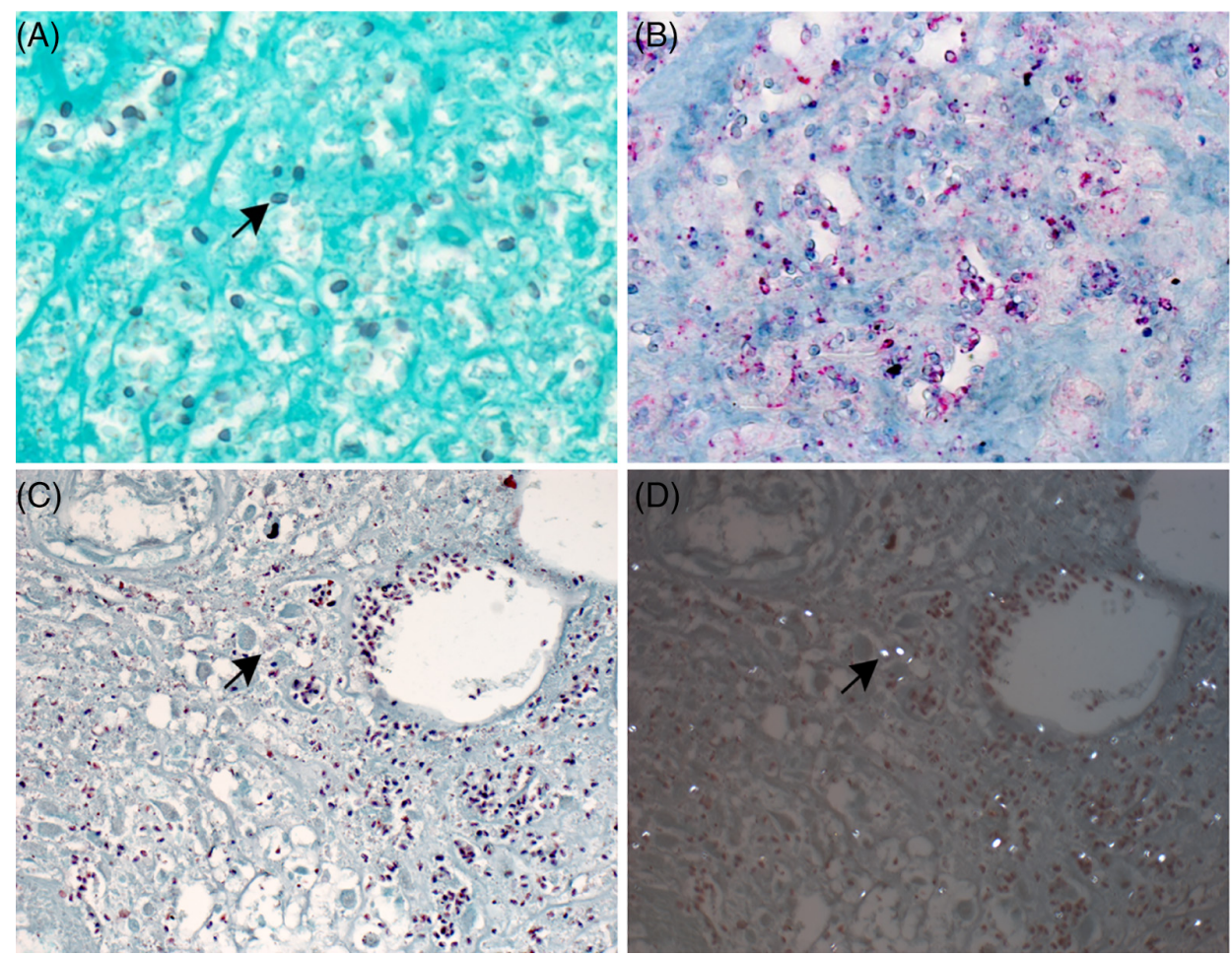
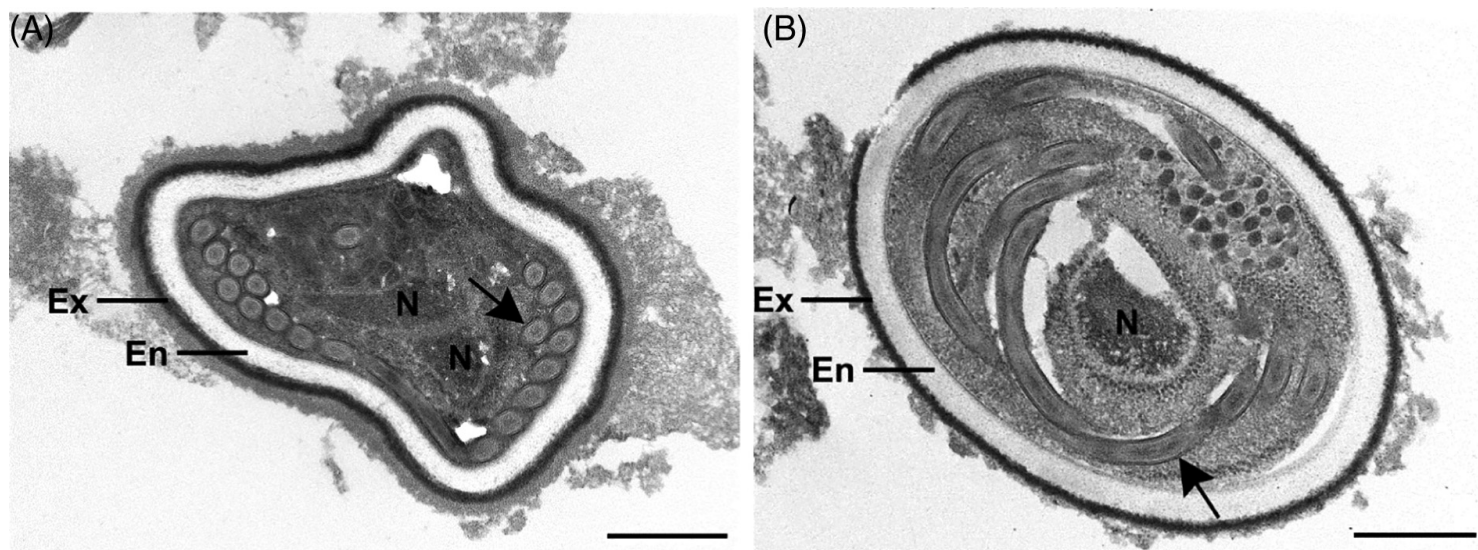

FIGURE 4 A and B, Electron microscopic images of Anncaliia algerae spores. Note cross sections of the polar tubule (arrows) in two rows in an anisofilar arrangement, which allowed for confirmation of microsporidia species. N, nucleus. Ex, exospore. En, endospore. Bars, $0.5 \mu \mathrm{m}$

superficial and deep lymphocytic and granulomatous dermatitis with focal necrosis (Figure 2). Tissue stains for microorganisms revealed 1 to $3 \mu \mathrm{m}$, round to oval structures within the necrotic areas, best highlighted by Giemsa, Steiner, and Fite stains (not shown). Additional studies were performed at the Centers for Disease Control and Prevention $(C D C)$ and confirmed the presence of microsporidia species. A Gomori methenamine silver (GMS) stain (Figure 3A) and an immunohistochemical stain by using rabbit anti-Encephalitozoon cuniculi serum (Figure 3B) highlighted the spores. L-T Gram was also performed (Figure $3 \mathrm{C}$ ) and polarization of the slide revealed bright white spores (Figure 3D). Electron microscopy identified diplokaryotic organisms containing up to 12 coils of polar tubules in two rows in anisofilar arrangement (Figure 4A,B), confirming microsporidia species. Speciation was determined by PCR to be $A$. algerae, formerly known as Nosema and Brachiola.

Further questioning revealed that the patient experienced an episode of diarrhea 6 months prior to the onset of cutaneous lesions, which lasted $\sim 10$ days and resulted in a 25 -35-pound weight loss. She also had recurrent sinusitis throughout this time. She denied a history of travel preceding her symptoms. The patient reported exposure to domestic pets and wild animals near her home, representing the most probably infectious reservoir. We hypothesized that ingestion of spores resulted in primary gastroenteritis with subsequent hematogenous dissemination to the skin facilitated by immunosuppressive drugs. 
The patient was discontinued from mycophenolate mofetil and was placed on a 21-day course of albendazole, with improvement in her skin lesions. She returned to Indiana for her follow-up care.

\section{3 | DISCUSSION}

Microsporidia infection frequently begins with gastrointestinal manifestations, which may distort villous architecture and lead to diarrhea and malabsorption. ${ }^{1,2}$ In addition, microsporidia may disseminate from the intestinal tract and result in numerous other clinical manifestations, particularly in immunocompromised patients. These manifestations may include upper respiratory symptoms, acute kidney injury, encephalitis, myositis, and, in very rare cases, subcutaneous nodular disease. $^{1-4,6}$ Our patient's immunosuppression by mycophenolate mofetil probably predisposed her to microsporidia infection, most probably from the animals to which she was exposed. We believe this began with gastrointestinal symptoms, although her infection probably disseminated hematogenously early on, resulting in sinusitis-like symptoms and cutaneous nodules. The steroid taper, although initially resulting in mild improvement, probably worsened her disseminated disease state because of further immunosuppression. In fact, two prior reports describe worsening microsporidiosis with steroid use. ${ }^{4,10}$

To the best of our knowledge, only three other examples of microsporidial infection with nodular skin lesions have been reported in the literature. One of these cases occurred in a patient with latestage HIV and a history of chronic sinusitis. ${ }^{3}$ Another case occurred in a patient with chronic lymphocytic leukemia. ${ }^{11}$ As in our patient, microsporidia infection was originally not considered a likely etiology because initial tissue stains for microorganisms were negative for an infectious cause. In this previously published case, the subcutaneous nodules were later associated with adjacent osteomyelitis. This suggests that microsporidia organisms can be locally destructive, resulting in a severe infection. Therefore, cutaneous microsporidia infections should be diagnosed and treated promptly in order to prevent such a harmful sequela. Both this report and our case illustrate the difficulty in identifying microsporidia on conventional staining. Immunohistochemical stains for microorganisms and electron microscopy have been shown to be highly effective and definitive in identifying these organisms in a timely manner. ${ }^{8,9,12}$ DNA amplification studies are also important in confirming infection. ${ }^{13}$

Another report of subcutaneous nodules associated with microsporidia infection occurred in a patient who was immunosuppressed with fludarabine, cyclophosphamide, alemtuzumab, and cyclosporine after undergoing an autologous stem cell transplant for multiple myeloma. ${ }^{4}$ This patient experienced pulmonary manifestations in addition to cutaneous findings. As in the case of our patient, the microsporidia organisms were initially not seen on staining. The patient was treated with systemic steroids, which resulted in initial mild improvement but eventual worsening of the infection. ${ }^{4}$ The patient ultimately died of pulmonary-related manifestations. Similar to our case, this example highlights the difficulty in diagnosing microsporidia infection and the risks of inadvertently using steroids to manage such cases.
Further speciation of organisms in these two prior cases of cutaneous microsporidia disease revealed $E$. intestinalis in the patient with $\mathrm{HIV}^{14}$ and Tubulinosema acridophagus in the patient with multiple myeloma. ${ }^{4}$ Neither of these organisms was present in our patient; instead, A. algerae was confirmed by PCR. A. algerae infection has been shown to be associated with severe myositis in immunocompromised patients ${ }^{15-17}$; however, to date, no examples of subcutaneous nodules secondary to $A$. algerae microsporidial infection have been described. One example of a skin abscess associated with A. algerae infection was reported in an immunosuppressed patient receiving chemotherapy; however, diffuse nodular disease, as was present in our patient, has not been reported. ${ }^{18}$

Although albendazole is the treatment of choice in patients with most species of microsporidial infections, specific efficacy against A. algerae has not been studied. ${ }^{13,15}$ Our patient's skin lesions improved significantly with albendazole, suggesting that it is indeed an effective treatment option for A. algerae.

Our case illustrates the benefit of taking a full review of systems and complete history in immunocompromised patients with skin diseases. The fact that this patient had exposure to animals along with an extensive history of diarrhea and sinusitis proved to be key information in identifying potential etiologies of her disease. This case report is extraordinary in that it describes one of only a handful of cases of confirmed cutaneous microsporidiosis. It also emphasizes the difficulty in diagnosing systemic microsporidial disease. It highlights the fact that the use of multiple different histochemical stains can assist with the identification of these microorganisms; once identified, uniformity in size and structure is highly suggestive of the presence of microorganisms. Ancillary studies, such as immunohistochemistry, electron microscopy, and PCR can be helpful in confirming the diagnosis and aid the clinician in selecting the appropriate treatment for the patient. Disseminated microsporidia infection should be considered as a possible differential diagnosis in all immunocompromised patients with skin nodules and recent gastrointestinal disease.

\section{ACKNOWLEDGMENTS}

The findings and conclusions in this report are those of the authors and do not necessarily represent the official position of the Centers for Disease Control and Prevention.

\section{ORCID}

Daniel A. Nadelman (iD https://orcid.org/0000-0002-7953-6240

Douglas R. Fullen (D) https://orcid.org/0000-0002-2524-4509

\section{REFERENCES}

1. Field AS, Milner DA. Intestinal microsporidiosis. Clin Lab Med. 2015; 35(2):445-459.

2. Nagpal A, Pritt BS, Lorenz EC, et al. Disseminated microsporidiosis in a renal transplant recipient: case report and review of the literature. Transpl Infect Dis. 2013;15(5):526-532.

3. Kester KE, Turiansky GW, McEvoy PL. Nodular cutaneous microsporidiosis in a patient with AIDS and successful treatment with longterm clindamycin therapy. Ann Intern Med. 1998;128(11):911-914. 
4. Meissner EG, Bennett JE, Qvarnstrom Y, et al. Disseminated microsporidiosis in an immunosuppressed patient. Emerg Infect Dis. 2012; 18(7):1155-1158.

5. Weber R, Bryan RT, Schwartz DA, Owen RL. Human microsporidial infections. Clin Microbiol Rev. 1994;7(4):426-461.

6. Didier ES, Weiss LM. Microsporidiosis: not just in AIDS patients. Curr Opin Infect Dis. 2011;24(5):490-495.

7. Garcia LS. Laboratory identification of the microsporidia. J Clin Microbiol. 2002;40(6):1892-1901.

8. Hocevar SN, Paddock CD, Spak CW, et al. Microsporidiosis acquired through solid organ transplantation: a public health investigation. Ann Internal Med. 2014;160(4):213-220.

9. Smith RM, Muehlenbachs A, Schaenmann J, et al. Three cases of neurologic syndrome caused by donor-derived microsporidiosis. Emerg Infect Dis. 2017;23(3):387-395.

10. Chan CM, Theng JT, Li L, Tan DT. Microsporidial keratoconjunctivitis in healthy individuals: a case series. Ophthalmology. 2003;110(7): 1420-1425.

11. Connors WJ, Carson JA, Chan WW, Parkins MD. Albendazoleresponsive disseminated Tubulinosema acridophagus in a patient with chronic lymphocytic leukemia. Clin Microbiol Infect. 2017;23(9): 684-685.

12. Shadduck JA. Human microsporidiosis and AIDS. Rev Infect Dis. 1989; 11(2):203-207.

13. De Groote MA, Visvesvara G, Wilson ML, et al. Polymerase chain reaction and culture confirmation of disseminated Encephalitozoon cuniculi in a patient with AIDS: successful therapy with albendazole. $J$ Infect Dis. 1995;171(5):1375-1378.

14. Kester KE, Visvesvara GS, McEvoy P. Organism responsible for nodular cutaneous microsporidiosis in a patient with AIDS. Ann Intern Med. 2000;133(11):925.

15. Watts MR, Chan RC, Cheong EY, et al. Anncaliia algerae microsporidial myositis. Emerg Infect Dis. 2014;20(2):185-191.

16. Field AS, Paik JY, Stark D, et al. Myositis due to the microsporidian Anncaliia (Brachiola) algerae in a lung transplant recipient. Transpl Infect Dis. 2012;14(2):169-176.

17. Coyle CM, Weiss LM, Rhodes LV 3rd, et al. Fatal myositis due to the microsporidian Brachiola algerae, a mosquito pathogen. N Engl J Med. 2004;351(1):42-47.

18. Cali A, Neafie R, Weiss LM, et al. Human vocal cord infection with the microsporidium Anncaliia algerae. J Eukaryot Microbiol. 2010;57 (6):562-567.

How to cite this article: Nadelman DA, Bradt AR,

Qvarnstrom Y, et al. Cutaneous microsporidiosis in an immunosuppressed patient. J Cutan Pathol. 2020;47(7):

659-663. https://doi.org/10.1111/cup.13674 